\title{
PARENTAL DECISIONS ON CHILDREN PARTICIPATING IN RESEARCH
}

\section{Open access}

Correspondence

mohamed.elemraid@ncl.ac.uk

Mohamed Elemraid is paediatric registrar and research fellow Kerry Pollard is paediatric research nurse

Matthew Thomas is paediatric registrar and research fellow Clare Simmister is paediatric research nurse

David Spencer is consultant respiratory paediatrician All at Great North Children's Hospital, Newcastle upon Tyne Hospitals NHS Foundation Trust, Newcastle upon Tyne, UK

Stephen Rushton is professor of biological modelling, school of biology, Newcastle University, Newcastle upon Tyne, UK

Andrew Gennery and Julia Clark are consultants in paediatric infectious disease and immunology at Great North Children's Hospital, Newcastle upon Tyne Hospitals NHS Foundation Trust, Newcastle upon Tyne, UK

Date of submission

September 262012

\section{Date of acceptance}

January 252013

\section{Peer review}

This article has been subject

to open peer review and

has been checked using

antiplagiarism software

\section{Mohamed Elemraid and colleagues consider what influences parents in allowing their child to be enrolled in clinical research}

\begin{abstract}
Aim To observe and report rates of, and reasons for, parents' refusal to consent to the participation of their children in appropriate clinical research.

Methods The parents of children admitted to hospital with a diagnosis of pneumonia or of empyema were asked for informed consent to research involving blood, urine and nasopharyngeal secretion samples from their child. Circumstances and numbers of agreements and refusals were compared and underlying reasons suggested.

Results Of 144 consent requests, ten were refused, which appeared to be linked to: not wanting the child to undergo further tests, lack of interest in participating in studies, research possibly delaying discharge, and
\end{abstract}

IT IS RECOGNISED that children are underrepresented in clinical research (McIntosh et al 2000). This has had an effect on the evidence base available for the management of sick children (Medical Research Council (MRC) 2004). The barriers to research in children are well documented (McIntosh et al 2000, Nabulsi et al 2011). One such barrier is the problem of obtaining informed consent (National Research Ethics Service (NRES) 2011). Assessing a child's ability to understand and make decisions about participation in research is a challenge and varies between individual children (MRC 2004). It is a legal requirement that, in children aged under 16 years, parents or guardians are asked to consent on their behalf before they can be enrolled in studies (MRC 2004, NRES 2011). anxiety regarding written consent and the length of information sheets.

Conclusions Severity of the child's illness appeared to determine the parent's decision. Involvement and assistance of non-research nursing and medical staff and previous introductions to the researchers are helpful. The timing and setting for the consent process should be selected carefully. Adequate, accessible study information for parents and children contributes to successful recruitment of participants.

\section{Keywords}

Informed consent, parents and guardians, research in children

International good clinical practice guidelines provide unified standards in research governance, which governments can adopt to protect the human rights of participants and also to define the roles of funders, investigators and monitors (International Conference on Harmonisation (ICH) 1996).

In a previous study of British parents consenting to an interventional trial, the major reasons for participation given were: benefit to other children in the future, contribution to science and benefit to their own child (Sammons et al 2007, Douglas et al 2011). Conversely, parents may feel an obligation to protect their children from potential harm or painful procedures (Sammons et al 2007, Nabulsi et al 2011). These factors are likely to influence the way in which parents rank the risks and potential benefits 
for their child, before making decisions regarding enrolment (Snethen et al 2006, Sammons et al 2007, Nabulsi et al 2011). Given the urgent need to increase the involvement of children in clinical research, greater understanding of the process of consent is vital so that the potential knowledge, attitudinal and psychosocial barriers to this procedure can be addressed (Chappuy et al 2008, John et al 2008, Truong et al 2011).

There is a considerable body of literature about children's consent to participation in research, particularly in clinical trials (Ross et al 1999, Douglas et al 2011, Truong et al 2011, Barakat et al 2012). However, most studies examine recruitment outcomes with minimal emphasis on strategies to improve the recruitment process (Blom-Hoffman et al 2009). Evidence from pooled review data show that barriers to participation in research, such as time constraints and demands of additional study procedures, are related to participants and researchers (Ross et al 1999).

Approaches such as taking time to establish rapport with children and their parents, short consent forms, the presence of the research team when discussing the study with families and giving them the opportunity to request further information were found to improve recruitment rates (Blom-Hoffman et al 2009). Several other factors described in the literature appear important: for example, trained research staff can significantly increase success in obtaining consent (Notter 1993, Hunt and White 1998), whereas complex information sheets and family difficulties in understanding the process can be significant barriers to consent (Sammons et al 2007).

Data were examined from two studies investigating childhood pneumonia and childhood empyema, respectively, conducted in the same setting, to identify potential reasons for parental consent refusal. The aim was also to report our experience of approaches that improved the recruitment procedures.

\section{Methods}

Study procedures Data were collected prospectively from two studies investigating the impact of the routine introduction of a pneumococcal conjugate vaccination programme in 2006 on the aetiology and epidemiology of childhood communityacquired pneumonia, and childhood empyema (Clarke 2006). These studies were undertaken at the Newcastle upon Tyne NHS Foundation Trust between April 2010 and March 2011. Informed consent was voluntarily obtained from a parent or guardian (person or authority who holds parental responsibility) (Office of the High Commissioner for Human Rights (OHCHR) 1989, ICH 1996, McIntosh et al 2000, MRC 2004). If the consent was refused, the following information was documented: reason for refusal if an explanation was offered, although parents were not asked to express their reasoning; who approached parents for consent; and the severity of pneumonia based on the national guidelines for the management of childhood pneumonia (British Thoracic Society 2002). In line with this guidance, all children with empyema were regarded as severely ill.

Children were eligible for the pneumonia study if aged 0-16 years and seen in hospital with clinical and radiological features of pneumonia. Similarly, children were eligible for the empyema study if they had clinical and radiological diagnosis of empyema and underwent pleural drainage.

Ethical considerations Favourable ethical opinions were obtained from the Newcastle and North Tyneside Research Ethics Committee for the pneumonia study and from the Sunderland Research Ethics Committee for the empyema study. Approval was not granted to ask parents about the refusal, hence they were not asked directly or systematically about declining the enrolment.

Recruitment process During the time of hospital admission, usually after the diagnosis had been confirmed, a member of the research team would visit the ward. This was either the research nurse (KP) or the research registrar (ME) for the pneumonia study, or the research nurse (CS) or the research registrar (MT) for the empyema study. Written information on the pneumonia study consisted of a four-page sheet for parents and separate two-page information documents for children under and over ten years of age, respectively. For the empyema study, a three-page parent information sheet and a three-page age-appropriate information document for the child were offered.

Before approaching the parents and child, liaison with medical and nursing staff helped to establish a suitable time to speak to them about the study. Requests for consent to the empyema study were generally delayed until the invasive procedures had been carried out. As all children with empyema were eligible for both studies, efforts were made to co-ordinate visits between the two separate research teams and thus avoid multiple requests to families. Study information was then presented verbally and in writing to the parents and, unless the parents specifically requested to give consent immediately, 
a suitable time was established to return and complete the consent process if they wished to join the study. The verbal explanation took up to ten minutes, after which parents were offered the opportunity to ask questions. Consents were obtained at the child's bedside. Where it was age-appropriate, children were involved in the consent process and they could themselves accept or decline the study.

If written consent was obtained then children with pneumonia had a standard proforma completed for epidemiological, clinical and management characteristics, together with samples of nasopharyngeal secretions, urine and blood. Three questions regarding medical history were asked of children with empyema, and a saliva sample was obtained either by expectoration or by use of soft-tipped oropharyngeal saliva swabs for those unable to expectorate.

\section{Results}

A total of 116 children were eligible for enrolment in the pneumonia study and 28 were also eligible for the empyema study, giving a total of 144 consent procedures. In all, ten (8 per cent) consents were refused, eight of which concerned children with severe pneumonia or empyema. Of the ten refusals, five had been seen by a research nurse and the remainder by a research registrar (Table 1). Of those with severe disease, consent refusals were 5 per cent and 11 per cent for pneumonia and empyema studies, respectively.

Refusal was linked to: not wanting the child to undergo further tests, lack of interest in participating in research, research possibly delaying discharge, and anxiety regarding written consent and the length of information sheets (Table 1). It was also observed that if the child and family were

\section{Table 1 Summary of consent refusals}

\begin{tabular}{|l|rl}
\hline Staff obtaining consent & \multicolumn{2}{|c}{ Refusals (n) (\%) } \\
\hline Research nurses & $5 / 68 \quad(7.3)$ \\
\hline Pneumonia study & $4 / 52$ & $(7.7)$ \\
\hline Empyema study & $1 / 16$ & $(6.2)$ \\
\hline Research registrars & $5 / 76$ & $(6.6)$ \\
\hline Pneumonia study & $3 / 64$ & $(4.7)$ \\
\hline Empyema study & $2 / 12(16.7)$ \\
\hline
\end{tabular}

approached before surgery, they would be reluctant to discuss their involvement in research at that time. About two days post-operatively, when the child became clinically stable, appeared the best time to discuss the study and consent.

Often parents declined the option to read the information and felt able to give consent based on the verbal information offered, together with discussion to answer questions they might have. None asked for more information on either of the studies; parents commented on the information sheets being long and complicated. Children were difficult to engage during the recruitment phase. Few were interested in the verbal explanation of the study and, although age-appropriate information was always offered, not many looked at or read it.

\section{Discussion}

This article reports consent refusal rates from two clinical studies, and describes the potential reasons contributing to the refusals. Although the acceptance rates for both studies were high, suggesting effective recruitment strategies, the severity of the child's illness appeared to influence parents' decisions on enrolment in clinical research. It was found that the willingness of research teams to provide parents with adequate study information, as well as the liaison of research team members with nursing and admitting medical staff about suitable times to approach families, contributed to successful recruitment.

Informed consent and assent The main elements of informed consent include adequate information, freedom of decision and capacity to understand that withdrawal can happen at any time without influencing treatment decision of patients (LoVerde et al 1989, ICH 1996, Nabulsi et al 2011). Informed consent must be sought from parents or legal guardians before enrolling children aged under 16 years in research in the UK, according to the research guidelines from the MRC (OHCHR 1989, ICH 1996, MRC 2004) and the European Ethics Working Group in Paediatrics (Gill et al 2003). Consent is given after written information sheets and verbal explanations have been provided.

The results of this research suggest that there is a balance between providing adequate information and offering over-lengthy information sheets that can often appear time-consuming and intrusive. In fact, one parent, after reading the information sheet, said it was too long and declined the study, despite earlier accepting it on verbal explanation. A onepage information sheet was initially created, but the ethics committees advised providing comprehensive 
information that covered essential facts about the studies. The essential information required for consent for research is governed by a legal and statutory framework and any changes can only be made within this framework (ICH 1996).

Assent is a concept that allows the participation of older competent children in the recruitment decision, which in turn encourages a sense of ownership and is defined as 'positive agreement' (Green et al 2003, Wendler 2006). Competence is considered not to depend primarily on age, but rather on the ability to understand and weigh up options (MRC 2004). In the UK, assent is advocated if the researcher feels that the child is able to make a decision about study participation (MRC 2004). It refers to 'acquiescence and affirmative agreement to participate' as defined by the Royal College of Paediatrics and Child Health and by the MRC (McIntosh et al 2000, MRC 2004).

Involving older children in the initial consent discussion is important, as the child needs to feel respected (Swartling et al 2011, Kumpunen et al 2012, Sibley et al 2012, Wilkinson 2012). Researchers should recognise that children are developing their autonomous decision-making capacity (Meaux and Bell 2001), albeit at varying rates. In practice, experience has shown that it is difficult to engage with children in general despite researchers' explanations (Unguru et al 2010) and there are concerns that the distinction between consent and assent is confusing (Baines 2011).

In this study, competent children were often observed not to be interested in verbal explanation or written information (Tait et al 2003a, 2003b). They frequently referred to their parents to sign the consent on their behalf. Hence in older competent children, as well as the assent form if provided, consent is obtained from their parents. This is unsurprising in the context of tired and ill children who may also be socially wary of new people (Blom-Hoffman et al 2009).

Taking into account how many families were approached by each member of the team (Table 1), the results showed the rates of refusal did not vary between different team members, suggesting that the role of the researcher who requested consent did not determine the likelihood of agreement to participation (Gibson et al 2011). Hence, the data gathered for this research support the increasing role of nurses in the field of research.

Having researchers join medical teams during ward rounds following admission and being introduced to the families were found to be helpful, and refusal was not noticed in such situations. This may indicate that admitting and research staff should appear as a single team. Co-ordination with the ward-based non-research nursing staff was useful in building bridges between families and researchers, as well as helping the research teams to collect study samples.

Timing and environment When a child is admitted to hospital with an illness and consent is sought soon after diagnosis, parents will be making decisions when they are stressed and vulnerable, while simultaneously trying to comfort their child (Shilling and Young 2009). The pneumonia and empyema research teams used approaches that accorded with the guidance on how to seek consent provided by the Royal College of Paediatrics and Child Health (McIntosh et al 2000).

In this research, parents were offered the study information sheets either before or at the time of interview for consent. If at the initial meeting with parents it was felt to be an inappropriate time to mention consent to research, in view of previous events or the child's condition or parental anxiety, information sheets were left with the parents and an appointment made to come back at a later time. It did not appear that more of these parents read the information than those given the information at time of interview. Parents reported not reading the information leaflets, and the information provided did not appear to influence the questions that were asked by them. This would reflect trust in the researcher obtaining consent, as previously reported (Chappuy et al 2010). Because of the nature of acute illness, when the admission period is often minimal, the process of obtaining informed consent usually occurred during one meeting.

Ideally, the process of informed consent should be presented in a relaxed and non-coercive environment (MRC 2004). Often, study information was delivered and consent obtained at the child's bedside. This allowed the parent to stay with their child and the child to continue with their activity should they not want to listen to the researcher.

However, confidentiality may be a consideration when the child is in a bay with other children and families. In the authors' experience with children with empyema or severe pneumonia, timing is as important as the environment chosen when gaining consent, highlighting the challenges when seeking consent for severely unwell children (Maitland et al 2011). It was found that if the child and family were approached before decortication surgery, they would be reluctant to discuss their involvement in research at that time as parents would often feel distressed and vulnerable while simultaneously trying to comfort their sick child. About two days 
post-operatively, when the child was in a less critical condition, appeared to be the best moment for obtaining consent and related specimens. It was notable that in the documented seven refusals among children with pneumonia, five had severe disease. It could be deduced that when parents' anxiety and their desire to protect the child from further harm are intensified, refusal may be one result.

Limitations Parents were not directly asked why they did not want to participate. This could affect the conclusions on factors influencing consent. The research team observed that parents' response and their own personal experience were subject to self-report bias. These limitations highlight the tensions and barriers involved in trying to improve the consent process and that information sheets had to be offputtingly long, and confidentiality in a ward bay was a problem.

\section{Conclusion}

Reasons for refusal to enrol children in research are complex, and are influenced by illness severity and study procedures. Consent forms should be simple for easy understanding. These findings suggest that increasing the research awareness and involvement of non-research nursing and medical staff members is important for improving the recruitment outcomes in clinical research. Therefore this warrants a systematic evaluation of the role of non-research departmental staff in recruitment procedures.

\section{Implications for practice}

Consent forms should be simple so that they can be understood easily.

- Involvement and assistance of non-research nursing and medical staff are important.

- Previous introduction of the researchers to the family is helpful.

- The timing and setting for the consent process should be carefully chosen as appropriate to the child, their parents and the circumstances.

Confidentiality, respect and empathy should be maintained.

The participation of competent older children in the recruitment decision is desirable.

\section{Conflict of interest}

The pneumonia study was funded with a grant from Pfizer Vaccines UK. The empyema study was supported by grants from Pfizer and GlaxoSmithKline Biologicals Belgium. The sponsors had no role in the study design, data collection, analysis or interpretation. JC and DS received unconditional research grants from Pfizer, and GSK for DS. JC and DS were the chief investigators respectively for the pneumonia and empyema studies

\section{Acknowledgement}

The authors are grateful to the paediatric staff at the Newcastle upon Tyne Hospitals NHS

Foundation Trust for help in enrolment and study procedures. Thanks to Helen Close, health research methodologist at Durham University, for reviewing the final manuscript

\section{References}

Baines P (2011) Assent for children's participation in research is incoherent an wrong. Archives of Disease in Childhood. 96, 10, 960-962.

Barakat LP et al (2012) Initial development of a questionnaire evaluating perceived benefits and barriers to pediatric clinical trials participation. Contemporary Clinical Trials. 34, 2, 218-226.

Blom-Hoffman J et al (2009) Consent procedures and participation rates in school-based intervention and prevention research: using a multi-component, partnership-based approach to recruit participants. School Mental Health. 1, 1, 3-15. British Thoracic Society (2002) Guidelines for the management of community acquired pneumonia in childhood. Thorax. 57, Suppl $1, \mathrm{i} 1-24$

Chappuy H et al (2008) Children's views on their involvement in clinical research. Pediatric Blood and Cancer. 50, 5, 1043-1046.

Chappuy H et al (2010) Parental

comprehension and satisfaction in informed consent in paediatric clinical trials: a prospective study on childhood leukaemia. Archives of Disease in Childhood. $95,10,800-804$.

Clarke SC (2006) Control of pneumococcal disease in the United Kingdom the start of a new era. Journal of Medical Microbiology. $55,8,975-980$.

Douglas SJ et al (2011) Exploration of the factors influencing parental decisions to allow their children to participate in clinical trials in paediatric infectious diseases. Archives of Disease in Childhood. 96, Suppl 1, A46-A47.
Gibson BE et al (2011) Assessment of children's capacity to consent for research: a descriptive qualitative study of researchers' practices. Journal of Medical Ethics. 37, 8, 504-509.

Gill D et al (2003) Guidelines for informed consent in biomedical research involving paediatric populations as research participants. European Journal of Pediatrics. $162,7-8,455-458$.

Green JB et al (2003) Putting the 'informed' into 'consent': a matter of plain language. Journal of Paediatrics and Child Health. 39, 9, 700-703.

Hunt JR, White E (1998) Retaining and tracking cohort study members. Epidemiologic Reviews. 20, 1, 57-70.

International Conference on Harmonisation (1996) ICH of Technical Requirements for Registration of Pharmaceuticals for Human Use. Guideline for Good Clinical Practice. tinyurl.com/mngruao (Last accessed: September 12 2013.)

John T et al (2008) Children's consent and paediatric research: is it appropriate for healthy children to be the decision-makers in clinical research? Archives of Disease in Childhood. 93, 5, 379-383.

Kumpunen S et al (2012) Practical approaches to seeking assent from children. Nurse Researcher. 19, 2, 23-27.

LoVerde ME et al (1989) Research consent forms: continued unreadability and increasing length. Journal of General Internal Medicine. 4, 5, 410-412.

Maitland K et al (2011) Use of deferred consent for severely ill children in a multi-centre phase III trial. Trials. 12, 90, 36.
McIntosh N et al (2000) Guidelines for the thical conduct of medical research involvin children. Royal College of Paediatrics, Child Health: Ethics Advisory Committee. Archives of Disease in Childhood. 82, 2, 177-182.

Meaux JB, Bell PL (2001) Balancing recruitment and protection: children as research subjects. Issues in Comprehensive Pediatric Nursing. 24, 4, 241-251.

Medical Research Council (2004) Medical Research Involving Children. tinyurl.com/5j2qv3 (Last accessed: September 19 2013.)

Nabulsi M et al (2011) Parental attitudes towards and perceptions of their children's participation in clinical research: a developing-country perspective. Journal of Medical Ethics. 37, 7, 420-423.

National Research Ethics Service (2011) Ethical Review Requirements. tinyurl.com/nbd9lsf (Last accessed: September 27 2013.)

Notter J (1993) Supporting the nurse as well as the patient. British Journal of Nursing. 2, 19 945.

Office of the High Commissioner for Human Rights (1989) Convention on the Rights of the Child. www2.ohchr.org/english/law/crc.htm (Last accessed: August 12012. )

Ross S et al (1999) Barriers to participation in randomised controlled trials: a systematic review. Journal of Clinical Epidemiology. 52, 12, 1143-1156.

Sammons HM et al (2007) What motivates British parents to consent for research? A questionnaire study. BMC Pediatrics. 7, 12, 36.
Shilling V, Young B (2009) How do parents experience being asked to enter a child in a randomised controlled trial? BMC Medical Ethics. doi: 10.1186/1472-6939-10-1.

Sibley A et al (2012) Assent is not consent Journal of Medical Ethics. 38, 1, 3.

Snethen JA et al (2006) Family patterns of decision-making in pediatric clinical trials. Research in Nursing and Health. 29, 3, 223-232. Swartling U et al (2011) "My parents decide if I can. I decide if I want to." Children's views on participation in medical research. Journal of Empirical Research on Human Research Ethics. $6,4,68-75$.

Tait AR et al (2003a) Do they understand? Part I: parental consent for children participating in clinical anesthesia and surgery research. Anesthesiology. 98, 3, 603-608.

Tait AR et al (2003b) Do they understand? Part II: assent of children participating in clinical anesthesia and surgery research. Anesthesiology. 98, 3, 609-614.

Truong TH et al (2011) Outcomes of informed consent among parents of children in cancer

clinical trials. Pediatric Blood and Cancer. 57, 6, 998-1004.

Unguru Y et al (2010) The experiences of children enrolled in pediatric oncology research: implications for assent. Pediatrics. 125, 4, e876-883.

Wendler DS (2006) Assent in paediatric research: theoretical and practical considerations. Journal of Medical Ethics. $32,4,229-234$

Wilkinson D (2012) Dissent about assent in paediatric research. Journal of Medical Ethics. $38,1,2$. 